of Medicine, New Haven, CT. Baseline and oral glucose-stimulated plasma glucose and insulin levels were similar in children and adult groups. A late fall in plasma glucose level at 3 - 5 hours after glucose ingestion stimulated a rise in plasma epinephrine, twice as high in children compared to adults. Hypoglycemic symptoms (shaky, sweaty, weak, or tachycardia) increased in children but not in adults, in association with the late fall in plasma glucose. P300 amplitude, a measure of cognitive function, was significantly reduced when glucose concentration was lowered to $75 \mathrm{mg} / \mathrm{dl}$ in children, but was preserved until the level fell to $54 \mathrm{mg} / \mathrm{dl}$ in adults. Children are more vulnerable to effects of hypoglycemia on cognitive function than are adults. (Jones TW et al. Enhanced adrenomedullary response and increased susceptibility to neuroglycopenia: Mechanisms underlying the adverse effects of sugar ingestion in healthy children. I Pediatr February 1995;126:171-7). (Reprints: William V Tamborlane MD, Department of Pediatrics, Yale University School of Medicine, 333 Cedar St, New Haven, CT 06510).

COMMENT. This study shows that consumption of glucose by healthy children may be followed by a fall in plasma glucose sufficient to induce hormonal changes and adverse behavioral and cognitive effects. The authors stress that their data do not prove a causative role for dietary sugar in children with hyperactivity. However, a balanced diet of protein, fat, and complex carbohydrate, to limit postprandial falls in glucose levels, should avoid symptoms associated with the enhanced adrenomedullary responsiveness demonstrated in healthy children.

Mild hypoglycemia $(60 \mathrm{mg} / \mathrm{dl})$ caused a significant decline in performance on a battery of cognitive tests in a study of adolescents with insulin-dependent diabetes mellitus at the University of Pittsburgh School of Medicine. Neither hyperglycemia, nor the rapid drop from acute hyperglycemia to euglycemia, affected symptoms, cognitive function, or counterregulatory hormone secretion. (Gschwend $S$ et al. Effects of acute hyperglycemia on mental efficiency and counterregulatory hormones in adolescents with insulin-dependent diabetes mellitus. I Pediatr Feb 1995;126:178-84).

\title{
COGNITIVE EFFECTS OF CRANIAL IRRADIATION
}

The effects of cranial irradiation on neuropsychological test performance, 9 months after diagnosis of acute lymphoblastic leukemia (ALL), were evaluated in 74 children aged 3 to 6 years included in the Children's Cancer Group cooperative treatment trials. Children who received cranial irradiation (18 Gy divided in 10 fractions) plus intrathecal methotrexate had significantly lower scores on the McCarthy Motor Scale and the Token Test of receptive language and auditory comprehension, when compared to children receiving intrathecal methotrexate alone. Performance of tests of general cognition, visual motor integration, and receptive language requiring verbal recognition and visual recognition (Peabody Picture Vocabulary Test-R) showed no differences among the treatment groups. (MacLean WE Jr et al, for the Children's Cancer Group. Neuropsychological effects of cranial irradiation in young children with acute lymphoblastic leukemia 9 months after diagnosis. Arch Neurol February 1995;52:156-160). (Reprints: Dr D Hammond, Children's Cancer Group, PO Box 60012, Arcadia, CA 91066).

COMMENT. In the past nine months issues of PNB have included two previous reports of the adverse effects of cranial irradiation in 
children treated for ALL. In a study from the Institute of Child Health, University of London, children who received a second course of cranial radiotherapy for relapsing LL suffered from neurologic deficits, growth impairment, ventricular enlargement on MRI, and impairments of tests of verbal comprehension, attention, and memory. Girls were affected more than boys. (Ped Neur Briefs June 1994;8:47). Hypoplasia of the cerebellar vermis and cognitive deficits involving visual-spatial-motor coordination and memory were reported at 9 year follow-up in 13 children who received 24 Gy cranial radiation and intrathecal methotrexate at the University of New Mexico and centers in Canada. (Ped Neur Briefs Nov 1994;8:82). The late morbidity associated with cranial radiotherapy has been recognized for some time. The Children's Cancer Group study demonstrates that cognitive deficits may become evident as early as 9 months after treatment, even with more moderate levels of irradiation. Whole-brain radiotherapy for brain tumors may also result in significant IQ deficits in children treated before age 7 . (Progress in Pediatric Neurology II, 1994, p199).

\section{LANGUAGE DOMINANCE WITH LEFT HEMISPHERE TUMORS}

Hemispheric language dominance in 12 children with slow growing tumors near left hemisphere language areas was studied at the Cleveland Clinic, OH. Complex partial seizures began at or under 6 years of age. Tumor resection was performed at ages 5 to 20 years, an average of 8 years after seizure onset. Neurologic exam was normal in 11 patients. Ten $(83 \%)$ had amobarbital tests indicating left hemisphere language dominance, confirmed by cortical stimulation in 6 . Eleven with complete tumor resection were seizure-free at follow-up. Most had developmental neoplasms, often congenital. Paired $t$ tests found no differences between pre- and postoperative performance of language tests, and a tendency for improved language function after operation. (DeVos KJ et al. Language dominance in patients with early childhood tumors near left hemisphere language areas. Neurology February 1995;45:349-356). (Reprints: Dr Elaine Wyllie, Pediatric Epilepsy Program, The Cleveland Clinic Foundation, 9500 Euclid Ave, Cleveland, OH 44195).

COMMENT. Language dominance does not usually transfer to the contralateral hemisphere in young children with low-grade left frontal and temporal tumors. Successful tumor resection is accomplished by using cortical stimulation studies and sparing of the language areas in close proximity to the tumor.

\section{NEUROMUSCULAR DISORDERS}

\section{PYRIDOXINE-INDUCED SENSORY NEUROPATHY}

An 18-year-old man with seizures from birth was followed in the Department of Clinical Neurological Sciences, University of Western Ontario, London, and was found to have developed a sensory neuropathy by 2 years of age following treatment with pyridoxine in doses up to $2000 \mathrm{mg} /$ day. The initial seizure at birth responded to pyridoxine $150 \mathrm{mg} \mathrm{IV}$, after treatment with diazepam had failed. A sister had died in status epilepticus at age 9 days and had not received pyridoxine. Complex febrile seizures from 1 to 4 years, followed by recurrent afebrile convulsions, and at 13 years, complex partial seizures continued despite pyridoxine $2000 \mathrm{mg} /$ day, phenytoin, and phenobarbital. At 